\title{
Rare $\Lambda_{b} \rightarrow \Lambda \mathbf{l}^{+} \mathbf{l}^{-}$Decays with Polarized $\Lambda$
}

\author{
Chuan-Hung Chen ${ }^{a}$ and C. Q. Geng ${ }^{b}$ \\ ${ }^{a}$ Department of Physics, National Cheng Kung University \\ Tainan, Taiwan, Republic of China \\ ${ }^{b}$ Department of Physics, National Tsing Hua University \\ Hsinchu, Taiwan, Republic of China
}

\begin{abstract}
We investigate the rare baryonic exclusive decays of $\Lambda_{b} \rightarrow \Lambda l^{+} l^{-}(l=e, \mu, \tau)$ with polarized $\Lambda$. Under the approximation of the heavy quark effective theory (HQET), in the standard model we derive the differential decay rates and various polarization asymmetries by including lepton mass effects. We find that with the long-distance effects the decay branching ratios are $5.3 \times 10^{-5}$ for $\Lambda_{b} \rightarrow \Lambda l^{+} l^{-}(l=e, \mu)$ and $1.1 \times 10^{-5}$ for $\Lambda_{b} \rightarrow \Lambda \tau^{+} \tau^{-}$. The effects of new physics in the decay rates are also discussed. The integrated longitudinal $\Lambda$ polarizations are -0.31 and -0.12 , while that of the normal ones 0.02 and 0.01 , for di-muon and tau modes, respectively. The CP-odd transverse polarization of $\Lambda$ is zero in the standard model but it is expected to be sizable in some theories with new physics.
\end{abstract}




\section{Introduction}

It is known that the recent interest in flavor physics has been focused in the rare decays related to $b \rightarrow s l^{+} l^{-}$induced by the flavor changing neutral current (FCNC) due to the CLEO measurement of the radiative $b \rightarrow s \gamma$ decay [1]. In the standard model, these rare decays occur at loop level and provide us information on the parameters of the CabibboKobayashi-Maskawa (CKM) matrix elements [2] as well as various hadronic form factors. In the literature, most of studies have been concentrated on the corresponding exclusive rare B meson decays such as $B \rightarrow K^{(*)} l^{+} l^{-}$[3].

In this paper, we investigate the baryon decays of $\Lambda_{b} \rightarrow \Lambda l^{+} l^{-}$with $\Lambda$ being polarized. Unlike mesonic decays, the baryonic decays could maintain the helicity structure of interactions in transition matrix elements. Through this property, we will show that the polarization asymmetries of $\Lambda$ are sensitive to right-handed couplings which are suppressed in the standard model. Thus, these baryonic decays could be used to search for physics beyond the standard model.

To study the exclusive bayonic decays, one of the most difficulties is to evaluate the hadronic matrix elements. It is known that there are many form factors for the matrix elements of $\Lambda_{b}$ to $\Lambda$, which are hard to be calculated since they are related to the nonperturbative effect of QCD. However, in heavy particle decays, HQET could reduce the number of form factors and supply the information with respect to their relative size. In our numerical calculations, we shall use the results in HQET. It is also know that a large theoretical uncertainty in our calculation to the decays arises from the long-distance (LD) effect. To reduce the uncertainty, we shall study various kinematic regions to distinguish the LD contributions. In our calculations, as a completeness, we will include the lepton mass, which is important for the tau lepton mode.

The paper is organized as follows. In Sec. 2, we study the effective Hamiltonian for the di-lepton decays of $\Lambda_{b} \rightarrow \Lambda l \bar{l}$ and form factors in the $\Lambda_{b} \rightarrow \Lambda$ transition. In Sec. 3, we derive the general forms of the differential decay rates and the $\Lambda$ polarizations. In Sec. 4 , we give the numerical analysis. We present our conclusions in Sec. 5.

\section{Effective Hamiltonian and Form factors}

The effective Hamiltonian for the inclusive decay of $b \rightarrow s l^{+} l^{-}$is given by

$$
\mathcal{H}=-4 \frac{G_{F}}{\sqrt{2}} V_{t b} V_{t s}^{*} \sum_{i=1}^{10} C_{i}(\mu) O_{i}(\mu)
$$

where the expressions for the renormalized Wilson coefficients $C_{i}(\mu)$ and operators $O_{i}(\mu)$ can be found in Ref. [4]. In terms of the Hamiltonian in Eq. (四), the decay amplitude is written as

$$
\begin{aligned}
\mathcal{M}= & \frac{G_{F} \alpha_{e m}}{\sqrt{2} \pi} V_{t b} V_{t s}^{*}\left[\bar{s}\left(C_{9}^{e f f}(\mu) \gamma_{\mu} P_{L}-\frac{2 m_{b}}{q^{2}} C_{7}(\mu) i \sigma_{\mu \nu} q^{\nu} P_{R}\right) b \bar{l} \gamma^{\mu} l\right. \\
& \left.+\bar{s} C_{10} \gamma_{\mu} P_{L} b \bar{l} \gamma^{\mu} \gamma_{5} l\right]
\end{aligned}
$$

with $P_{L(R)}=\left(1 \mp \gamma_{5}\right) / 2$. We note that in Eq. (2), only the term associated with Wilson coefficient $C_{10}$ is independent of the $\mu$ scale. We also note that the dominant contribution to 


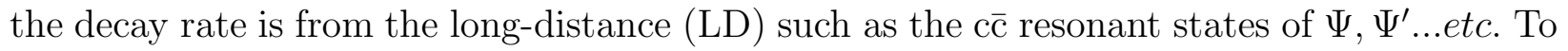
find out the LD effects for the B-meson decays, in the literature [5, 6, 7, 8, 9, 10, both the factorization assumption (FA) and the vector meson dominance (VMD) approximation have been used. For the LD contributions in baryonic decays, we assume that the parametrization is the same as that in the B meson decays. Hence, we include the resonant effects (RE) by absorbing it to the corresponding Wilson coefficients. The effective Wilson coefficient of $C_{9}^{e f f}$ has the standard form

$$
C_{9}^{e f f}=C_{9}(\mu)+\left(3 C_{1}(\mu)+C_{2}(\mu)\right)\left(h(x, s)+\frac{3}{\alpha_{e m}^{2}} \sum_{j=\Psi, \Psi^{\prime}} k_{j} \frac{\pi \Gamma\left(j \rightarrow l^{+} l^{-}\right) M_{j}}{q^{2}-M_{j}^{2}+i M_{j} \Gamma_{j}}\right),
$$

where $h(x, s)$ describes the one-loop matrix elements of operators $O_{1}=\bar{s}_{\alpha} \gamma^{\mu} P_{L} b_{\beta} \bar{c}_{\beta} \gamma_{\mu} P_{L} c_{\alpha}$ and $O_{2}=\bar{s} \gamma^{\mu} P_{L} b \bar{c} \gamma_{\mu} P_{L} c$ as shown in Ref. [四], $M_{j}$ and $\Gamma_{j}$ are the masses and widths of intermediate states, and the factors $k_{j}$ are phenomenological parameters for compensating the approximations of FA and VMD and reproducing the correct branching ratios of $B\left(\Lambda_{b} \rightarrow\right.$ $\left.\Lambda J / \Psi \rightarrow \Lambda l^{+} l^{-}\right)=B\left(\Lambda_{b} \rightarrow \Lambda J / \Psi\right) \times B\left(J / \Psi \rightarrow l^{+} l^{-}\right)$when we study the $\Lambda_{b}$ decays. We note that by taking $k_{\Psi} \simeq-1 /\left(3 C_{1}+C_{2}\right)$ and $B\left(\Lambda_{b} \rightarrow \Lambda J / \Psi\right)=(4.7 \pm 2.8) \times 10^{-4}$, the $k_{j}$ factors in the $\Lambda_{b}$ case are almost the same as that in the B meson one. In this paper we take the Wilson coefficients at the scale of $\mu \sim m_{b} \sim 5.0 \mathrm{GeV}$ and their values are $C_{1}\left(m_{b}\right)=-0.226$, $C_{2}\left(m_{b}\right)=1.096, C_{7}\left(m_{b}\right)=-0.305, C_{9}\left(m_{b}\right)=4.186$, and $C_{10}\left(m_{b}\right)=-4.599$, respectively.

It is clear that one of the main theoretical uncertainties in studying exclusive decays arises from the calculation of form factors. In general there are many form factors in exclusive baryon decays. However, the number of the form factors can be reduced by the heavy quark effective theory (HQET). With HQET, the hadronic matrix elements for the heavy baryon decays could be parametrized as follows [11]

$$
<\Lambda(p, s)|\bar{s} \Gamma b| \Lambda_{b}\left(v, s^{\prime}\right)>=\bar{u}_{\Lambda}(p, s)\left\{F_{1}(p \cdot v)+\not v F_{2}(p \cdot v)\right\} \Gamma u_{\Lambda_{b}}\left(v, s^{\prime}\right)
$$

with $R=F_{2}(p . v) / F_{1}(p . v)$ where $v$ is the four-velocity of heavy baryon and $\Gamma$ denotes the possible Dirac matrix. Note that in terms of HQET there are only two independent form factors in Eq. (团) for each $\Gamma$. In the following, we shall adopt the HQET approximation to analyze the behavior of $\Lambda_{b} \rightarrow \Lambda l^{+} l^{-}$.

\section{Differential Decay Rate and Polarizations}

In this section we present the formulas for the differential decay rates and the longitudinal and normal $\Lambda$ polarizations of $\Lambda_{b}\left(p_{\Lambda_{b}}\right) \rightarrow \Lambda\left(p_{\Lambda}, s\right) l^{+}\left(p_{l^{+}}\right) l^{-}\left(p_{l^{-}}\right)$. In our calculations, we have included the lepton masses. To study the $\Lambda$ spin polarization, we write the $\Lambda$ four-spin vector in terms of a unit vector, $\hat{\xi}$, along the $\Lambda$ spin in its rest frame, as

$$
s_{0}=\frac{\vec{p}_{\Lambda} \cdot \hat{\xi}}{M_{\Lambda}}, \quad \vec{s}=\hat{\xi}+\frac{s_{0}}{E_{\Lambda}+M_{\Lambda}} \vec{p}_{\Lambda}
$$

and choose the unit vectors along the longitudinal, normal, transverse components of the $\Lambda$ polarization, to be

$$
\hat{e}_{L}=\frac{\vec{p}_{\Lambda}}{\left|\vec{p}_{\Lambda}\right|},
$$




$$
\begin{aligned}
\hat{e}_{N} & =\frac{\vec{p}_{\Lambda} \times\left(\vec{p}_{l^{-}} \times \vec{p}_{\Lambda}\right)}{\left|\vec{p}_{\Lambda} \times\left(\vec{p}_{l^{-}} \times \vec{p}_{\Lambda}\right)\right|}, \\
\hat{e}_{T} & =\frac{\vec{p}_{l^{-}} \times \vec{p}_{\Lambda}}{\left|\vec{p}_{l^{-}} \times \vec{p}_{\Lambda}\right|}
\end{aligned}
$$

respectively. The partial decay width for $\Lambda_{b} \rightarrow \Lambda l^{+} l^{-}(l=e$ or $\mu$ or $\tau)$ is given by

$$
\begin{aligned}
d \Gamma= & \frac{1}{4 M_{\Lambda_{b}}}|\mathcal{M}|^{2}(2 \pi)^{4} \delta\left(p_{\Lambda_{b}}-p_{\Lambda}-p_{l^{+}}-p_{l^{-}}\right) \\
& \times \frac{d \vec{p}_{\Lambda}}{(2 \pi)^{3} 2 E_{\Lambda}} \frac{d \vec{p}_{l^{+}}}{(2 \pi)^{3} 2 E_{1}} \frac{d \vec{p}_{l^{-}}}{(2 \pi)^{3} 2 E_{2}}
\end{aligned}
$$

with

$$
|\mathcal{M}|^{2}=\frac{1}{2}\left|\mathcal{M}^{0}\right|^{2}\left[1+\left(P_{L} \hat{e}_{L}+P_{N} \hat{e}_{N}+P_{T} \hat{e}_{T}\right) \cdot \hat{\xi}\right]
$$

where $\left|\mathcal{M}^{0}\right|^{2}$ is related to the decay rate for the unpolarized $\Lambda$ and $P_{i}(i=L, N, T)$ denote the longitudinal, normal and transverse polarizations of $\Lambda$, respectively. Introducing dimensionless variables of $\lambda_{t}=V_{t b} V_{t s}^{*}, \hat{t}=E_{\Lambda} / M_{\Lambda_{b}}, r=M_{\Lambda}^{2} / M_{\Lambda_{b}}^{2}, \hat{m}_{l}=m_{l} / M_{\Lambda_{b}}, \hat{m}_{b}=m_{b} / M_{\Lambda_{b}}$ and $\hat{s}=1+r-2 \hat{t}$, and integrating the angle dependence of the lepton, the differential decay width in Eq. (17) can be rewritten as

$$
\begin{aligned}
d \Gamma & =\frac{1}{2} d \Gamma^{0}[1+\vec{P} \cdot \hat{\xi}] \\
d \Gamma^{0} & =\frac{G_{F}^{2} \alpha_{e m}^{2}\left|\lambda_{t}\right|^{2}}{192 \pi^{5}} M_{\Lambda_{b}}^{5} \sqrt{\left(\hat{t}^{2}-r\right)\left(1-\frac{4 \hat{m}_{l}^{2}}{\hat{s}}\right)} \rho_{0}(\hat{t}) d \cos \theta_{\Lambda} d \hat{t}
\end{aligned}
$$

with

$$
\vec{P}=P_{L} \hat{e}_{L}+P_{N} \hat{e}_{N}+P_{T} \hat{e}_{T}
$$

and

$$
\rho_{0}(\hat{t})=\left(\Gamma_{1}+\Gamma_{2}+\Gamma_{3}+\Gamma_{4}\right)
$$

where

$$
\begin{aligned}
\Gamma_{1}= & 4 \frac{\hat{m}_{b}^{2}}{\hat{s}}\left|C_{7}\right|^{2}\left\{-\left(F_{1}^{2}-F_{2}^{2}\right)(\hat{s} \hat{t}-4(1-\hat{t})(\hat{t}-r))\right. \\
& -2 F_{2}\left(F_{1} \sqrt{r}+F_{2} \hat{t}\right)\left(\hat{s}-4(1-\hat{t})^{2}\right)+8 \frac{\hat{m}_{l}^{2}}{\hat{s}}\left(\left(F_{1}^{2}-F_{2}^{2}\right)(1-\hat{t})(\hat{t}-r)\right. \\
& \left.\left.+2 F_{2}\left(F_{1} \sqrt{r}+F_{2} \hat{t}\right)(1-\hat{t})^{2}\right)-2 \hat{m}_{l}^{2}\left(\left(F_{1}^{2}+F_{2}^{2}\right) \hat{t}+2 F_{1} F_{2} \sqrt{r}\right)\right\} \\
\Gamma_{2}= & 12 \hat{m}_{b} \operatorname{Re} C_{9}^{e f f} C_{7}^{*}\left(1+2 \frac{\hat{m}_{l}^{2}}{\hat{s}}\right)\left[\left(F_{1}^{2}-F_{2}^{2}\right)(\hat{t}-r)+2 F_{2}\left(F_{1} \sqrt{r}+F_{2} \hat{t}\right)(1-\hat{t})\right], \\
\Gamma_{3}= & \left(\left|C_{9}^{e f f}\right|^{2}+\left|C_{10}\right|^{2}\right)\left\{\left(1-4 \frac{\hat{m}_{l}^{2}}{\hat{s}}\right) \hat{s}\left[\left(F_{1}^{2}+F_{2}^{2}\right) \hat{t}+2 F_{1} F_{2} \sqrt{r}\right]\right. \\
& \left.+2\left(1+2 \frac{\hat{m}_{l}^{2}}{\hat{s}}\right)(1-\hat{t})\left[(\hat{t}-r)\left(F_{1}^{2}-F_{2}^{2}\right)+2 F_{2}\left(F_{1} \sqrt{r}+F_{2} \hat{t}\right)(1-\hat{t})\right]\right\} \\
\Gamma_{4}= & 6 \hat{m}_{l}^{2}\left(\left|C_{9}^{e f f}\right|^{2}-\left|C_{10}\right|^{2}\right)\left[\left(F_{1}^{2}+F_{2}^{2}\right) \hat{t}+2 F_{1} F_{2} \sqrt{r}\right] .
\end{aligned}
$$


Here the form factors and Wilson coefficients in Eq. (12) depend on the $\Lambda$ energy $\left(E_{\Lambda}\right)$ and the scale of $\mu$. The ranges of $\hat{t}$ and $\hat{s}$ are as follows:

$$
\begin{aligned}
\sqrt{r} & \leq \hat{t} \leq \frac{1}{2}\left(1+r-4 \hat{m}_{l}^{2}\right), \\
4 \hat{m}_{l}^{2} & \leq \hat{s} \leq(1-\sqrt{r})^{2} .
\end{aligned}
$$

We note that our result for the differential decay rate in Eq. (9) is consistent with that given in Refs. 12, 13 when one takes the limit of massless lepton.

The longitudinal, normal and transverse $\Lambda$ polarization asymmetries in Eq. (8) can be defined by

$$
P_{i}(\hat{t})=\frac{d \Gamma\left(\hat{e}_{i} \cdot \hat{\xi}=1\right)-d \Gamma\left(\hat{e}_{i} \cdot \hat{\xi}=-1\right)}{d \Gamma\left(\hat{e}_{i} \cdot \hat{\xi}=1\right)+d \Gamma\left(\hat{e}_{i} \cdot \hat{\xi}=-1\right)} .
$$

From Eqs. (9) and (14), we obtain the polarizations of $P_{L}$ and $P_{N}$ to be

$$
P_{L}(\hat{t})=\frac{\sqrt{t^{2}-r}}{\sqrt{r} \rho_{0}(\hat{t})} D_{L}
$$

and

$$
\begin{aligned}
P_{N}(\hat{t})= & \frac{-3}{2 \rho_{0}(\hat{t})} \pi \sqrt{1-4 \frac{\hat{m}_{l}^{2}}{\hat{s}}} \sqrt{\hat{s}}\left[\left(F_{1}^{2}+F_{2}^{2}\right) \sqrt{r}+2 F_{1} F_{2} \hat{t}\right] \\
& \times\left[\operatorname{Re} C_{9}^{e f f} C_{10}^{*}(1-\hat{t})+2 \hat{m}_{b} \operatorname{Re} C_{10} C_{7}^{*}\right],
\end{aligned}
$$

respectively, where $D_{L}=L_{1}+L_{2}+L_{3}+L_{4}$ with

$$
\begin{aligned}
L_{1}= & -4 \frac{\hat{m}_{b}^{2}}{\hat{s}}\left(1-2 \frac{\hat{m}_{l}^{2}}{\hat{s}}\right)\left|C_{7}\right|^{2} \sqrt{r}\left\{-\left(1-4 \frac{\hat{m}_{l}^{2}}{\hat{s}}\right)\left(F_{1}^{2}-F_{2}^{2}\right) \hat{s}\right. \\
& \left.+4\left(1-\frac{\hat{m}_{l}^{2}}{\hat{s}}\right)\left(F_{1}^{2}-F_{2}^{2}+2 F_{2}^{2} \hat{t}+2 F_{1} F_{2} \sqrt{r}\right)(1-t)\right\} \\
& +8 \frac{\hat{m}_{l}^{2} \hat{m}_{b}^{2}}{\hat{s}}\left|C_{7}\right|^{2} \sqrt{r}\left\{\left(F_{1}^{2}+F_{2}^{2}\right)\left(1-10 \frac{1-\hat{t}}{\hat{s}}\right)+3\left(1-2 \frac{\hat{m}_{l}^{2}}{\hat{s}}\right)\left(F_{1}^{2}-F_{2}^{2}\right)\right. \\
& \left.-2\left(1-4 \frac{\hat{m}_{l}^{2}}{\hat{s}}\right) F_{2}^{2}+4\left(5-2 \frac{\hat{m}_{l}^{2}}{\hat{s}}\right)\left(\frac{1-\hat{t}}{\hat{s}}\right)\left(F_{2}^{2}(1-t)-F_{1} F_{2} \sqrt{r}\right)\right\}, \\
L_{2}= & -12 \hat{m}_{b} \operatorname{Re} C_{9}^{e f f} C_{7}^{*}\left(1+2 \frac{\hat{m}_{l}^{2}}{\hat{s}}\right) \sqrt{r}\left[\left(F_{1}^{2}-F_{2}^{2}\right)+2 \hat{t} F_{2}^{2}+2 \sqrt{r} F_{1} F_{2}\right], \\
L_{3}= & -\left(\left|C_{9}^{e f f}\right|^{2}+\left|C_{10}\right|^{2}\right) \sqrt{r}\left\{\left(1-4 \frac{\hat{m}_{l}^{2}}{\hat{s}}\right)\left(F_{1}^{2}-F_{2}^{2}\right) \hat{s}+2\left(1+2 \frac{\hat{m}_{l}^{2}}{\hat{s}}\right)(1-\hat{t}) \times\right. \\
& {\left[\left(F_{1}^{2}-F_{2}^{2}+2 \hat{t} F_{2}^{2}+2 F_{1} F_{2} \sqrt{r}\right]\right\}, } \\
L_{4}= & -6 \hat{m}_{l}^{2}\left(\left|C_{9}^{e f f}\right|^{2}-\left|C_{10}\right|^{2}\right)\left(F_{1}^{2}-F_{2}^{2}\right) \sqrt{r} .
\end{aligned}
$$

For the T-odd transverse $\Lambda$ polarization, we have that

$$
P_{T} \sim m_{s} \operatorname{Im} C_{10} C_{7}^{*}
$$


It is clear that $P_{T}$ is zero in the standard model since there is no phase in $C_{10} C_{7}^{*}$. We remark that even there is a phase in a theory of the standard model like, due to the suppression of $m_{s}, P_{T}$ is expected to be small. However, a possible CP violating right-handed interaction could induce a sizable $P_{T}$ [15]. Therefore, observing $P_{T}$ could indicate new physics beyond the standard model.

It is interesting to point out that we can also discuss $\Lambda_{b} \rightarrow \Lambda \bar{\nu} \nu$ by taking the limits of

$$
m_{l} \rightarrow 0, C_{7} \rightarrow 0, C_{9}^{e f f} \rightarrow \frac{X\left(x_{t}\right)}{\sin ^{2} \theta_{W}}, C_{10} \rightarrow-\frac{X\left(x_{t}\right)}{\sin ^{2} \theta_{W}}
$$

in Eqs. (7)-(18), where $X\left(x_{t}\right)=0.65 x_{t}^{0.575}$ [4] and $x_{t}=m_{t}^{2} / M_{W}^{2}$. Explicitly, we have

$$
\begin{aligned}
d \Gamma\left(\Lambda_{b} \rightarrow \Lambda \bar{\nu} \nu\right) & =\frac{1}{2} d \Gamma^{0}\left(\Lambda_{b} \rightarrow \Lambda \bar{\nu} \nu\right)\left[1+\vec{P}^{\nu \nu} \cdot \hat{\xi}\right] \\
d \Gamma^{0}\left(\Lambda_{b} \rightarrow \Lambda \bar{\nu} \nu\right) & =3 \frac{G_{F}^{2} \alpha_{e m}^{2} \lambda_{t}^{2}}{192 \pi^{5}} M_{\Lambda_{b}}^{5} \sqrt{\hat{t}^{2}-r} \rho^{\nu \nu}(\hat{t}) d \cos \theta_{\Lambda} d \hat{t}
\end{aligned}
$$

where

$$
\begin{aligned}
\rho^{\nu \nu}(\hat{t})= & 2\left(\frac{X\left(x_{t}\right)}{\sin ^{2} \theta_{W}}\right)^{2}\left\{\left[\left(F_{1}^{2}+F_{2}^{2}\right) \hat{t}+2 F_{1} F_{2} \sqrt{r}\right] \hat{s}+2(1-\hat{t})\right. \\
& \left.\times\left[(\hat{t}-r)\left(F_{1}^{2}-F_{2}^{2}\right)+2 F_{2}\left(F_{1} \sqrt{r}+F_{2} \hat{t}\right)(1-\hat{t})\right]\right\}
\end{aligned}
$$

and

$$
\begin{aligned}
P_{L}^{\nu \nu}(\hat{t})= & -2 \frac{\sqrt{t^{2}-r}}{\rho^{\nu \nu}(\hat{t})}\left(\frac{X\left(x_{t}\right)}{\sin ^{2} \theta_{W}}\right)^{2}\left\{\left(F_{1}^{2}-F_{2}^{2}\right) \hat{s}+2(1-t) \times\right. \\
& {\left[\left(F_{1}^{2}-F_{2}^{2}+2 \hat{t} F_{2}^{2}+2 F_{1} F_{2} \sqrt{r}\right]\right\} . }
\end{aligned}
$$

Here we have only listed the longitudinal polarization of $\Lambda$ because the momentum of the neutrino cannot be measured experimentally.

\section{Numerical Analysis}

In order to analyze the decay rate and polarization asymmetries, we use the Wilson coefficients at the scale $\mu \approx m_{b}$ as stated in Sec. 2. The other parameters used in our numerical calculations are listed in Table 1.

As to the $\Lambda_{b} \rightarrow \Lambda$ transition form factors, we adopt the results and input parameters given in Ref. [12], in which the QCD sum rule approach based on the framework of HQET was used. However, there is a undetermined parameter, Borel parameter $(\mathrm{M})$, in the approach, which is introduced to suppress the contribution from the higher excited and continuum states. According to the analysis of Ref. [12], it could be $1.5 \mathrm{GeV} \leq M \leq 1.9 \mathrm{GeV}$. For simplicity, we will take $M=1.7 \mathrm{GeV}$ in our numerical analysis. As a comparison, we will also present the results with the dipole form assumption [11].

\subsection{Decay Rates and Polarizations of $\Lambda$}

From Eqs. (9) and (12), by integrating the whole range of $\Lambda$ energy and setting phenomenological factor $\kappa=-1 /\left(3 C_{1}+C_{2}\right)$, the branching ratios of the dilepton decays are summarized in Table 2 and the distributions of the differential decay rates are shown in Figures 1 
Table 1: Input parameters used in our numerical calculations.

\begin{tabular}{|lll|}
\hline$M_{\Lambda_{b}}$ & 5.64 & $\mathrm{GeV}$ \\
$M_{\Lambda}$ & 1.116 & $\mathrm{GeV}$ \\
$m_{t}$ & 165 & $\mathrm{GeV}$ \\
$m_{b}$ & 4.8 & $\mathrm{GeV}$ \\
$m_{\tau}$ & 1.777 & $\mathrm{GeV}$ \\
$m_{\mu}$ & 1.05 & $\mathrm{GeV}$ \\
$m_{c}$ & 1.4 & $\mathrm{GeV}$ \\
$\alpha_{e m}$ & $1 / 129$ & \\
$\tau_{\Lambda_{b}}$ & $1.8848 \times 10^{12}$ & $\mathrm{GeV}^{-1}$ \\
$V_{t b} V_{t s}^{*}$ & 0.04 & \\
\hline
\end{tabular}

and 2 for $\Lambda_{b} \rightarrow \Lambda \mu^{+} \mu^{-}$and $\Lambda_{b} \rightarrow \Lambda \tau^{+} \tau^{-}$, respectively. Here we have also illustrated the results from the pole model [11]. The form factors with the dipole forms in the model are given by

$$
F_{1,2}\left(p_{\Lambda} \cdot v\right)=N_{1,2}\left(\frac{\Lambda_{Q C D}}{\Lambda_{Q C D}+p_{\Lambda} \cdot v}\right)^{2}
$$

where $p_{\Lambda} \cdot v=E_{\Lambda}$ and $\Lambda_{Q C D}$ is chosen to be around $200 \mathrm{MeV}$. From Eq. (23), one obtains that $R=F_{2} / F_{1}=N_{2} / N_{1} \sim-0.25$ [11, 14]. In terms of HQET the form factors of $\Lambda_{b} \rightarrow \Lambda$ should be the same as that of $\Lambda_{c} \rightarrow \Lambda$ at the maximal momentum transfer. Therefore, by using the measured branching ratio of $\Lambda_{c} \rightarrow \Lambda l \nu$, we extract that $\left|N_{1}\right| \sim 52.32$ with the same dipole forms.

Table 2: Decay branching ratios $(\mathrm{Br})$ based on the form factors from the QCD sum rule approach and the dipole model, respectively

\begin{tabular}{|c|c|c|c|c|c|}
\hline Model & Decay Br & $\Lambda_{b} \rightarrow \Lambda \nu \bar{\nu}$ & $\Lambda_{b} \rightarrow \Lambda e^{+} e^{-}$ & $\Lambda_{b} \rightarrow \Lambda \mu^{+} \mu^{-}$ & $\Lambda_{b} \rightarrow \Lambda \tau^{+} \tau^{-}$ \\
\hline \multirow{2}{*}{$\begin{array}{l}\text { QCD } \\
\text { Sum Rule }\end{array}$} & without LD & $1.6 \times 10^{-5}$ & $2.3 \times 10^{-6}$ & $2.1 \times 10^{-6}$ & $1.8 \times 10^{-7}$ \\
\hline & with LD & -- & $5.3 \times 10^{-5}$ & $5.3 \times 10^{-5}$ & $1.1 \times 10^{-5}$ \\
\hline \multirow[t]{2}{*}{ Pole Model } & without LD & $9.2 \times 10^{-6}$ & $1.2 \times 10^{-6}$ & $1.2 \times 10^{-6}$ & $2.6 \times 10^{-7}$ \\
\hline & with LD & -- & $3.6 \times 10^{-5}$ & $3.6 \times 10^{-5}$ & $9.0 \times 10^{-6}$ \\
\hline
\end{tabular}

From Table 2, we find that the branching ratios with including LD contributions are about $1-2$ orders of magnitude larger than that without LD ones and the results from the pole model are close to those from the QCD sum rule.

If it is not mentioned, we shall use the form factors from the QCD sum rule approach in the rest of our numerical analysis.

To estimate the contributions to the decay branching ratios by excluding the resonances of $J / \psi$ and $\psi^{\prime}$, we choose five separate regions in terms of the masses of $J / \psi$ and $\psi^{\prime}$, and they are given as follows

$$
I \quad: \quad M_{\Lambda} \leq E_{\Lambda} \leq\left. E\right|_{\max }-\delta_{\psi^{\prime}}^{1}
$$




$$
\begin{aligned}
I I & :\left.\quad E\right|_{\max }-\delta_{\psi^{\prime}}^{1} \leq E_{\Lambda} \leq\left. E\right|_{\max }-\delta_{\psi^{\prime}}^{2} \\
I I I & :\left.\quad E\right|_{\max }-\delta_{\psi^{\prime}}^{2} \leq E_{\Lambda} \leq\left. E\right|_{\max }-\delta_{J / \psi}^{1}, \\
I V & :\left.\quad E\right|_{\max }-\delta_{J / \psi}^{1} \leq E_{\Lambda} \leq\left. E\right|_{\max }-\delta_{J / \psi}^{2} \\
V & :\left.\quad E\right|_{\max }-\delta_{J / \psi}^{2} \leq E_{\Lambda} \leq\left. E\right|_{\max }
\end{aligned}
$$

where

$$
\begin{array}{ll}
\left.E\right|_{\max }=M_{\Lambda_{b}}\left(1+r-4 \hat{m}_{l}^{2}\right) / 2, & \\
\delta_{\psi^{\prime}}^{1}=\left(M_{\psi^{\prime}}+\sqrt{\sqrt{2} M_{\psi^{\prime}} \Gamma_{\psi^{\prime}}}\right)^{2} / 2 M_{\Lambda_{b}}, & \delta_{\psi^{\prime}}^{2}=\left(M_{\psi^{\prime}}-\sqrt{\sqrt{2} M_{\psi^{\prime}} \Gamma_{\psi^{\prime}}}\right)^{2} / 2 M_{\Lambda_{b}}, \\
\delta_{J / \psi}^{1}=\left(M_{J / \psi}+\sqrt{\sqrt{2} M_{J / \psi} \Gamma_{J / \psi}}\right)^{2} / 2 M_{\Lambda_{b}}, & \delta_{J / \psi}^{2}=\left(M_{J / \psi}-\sqrt{\sqrt{2} M_{J / \psi} \Gamma_{J / \psi}}\right)^{2} / 2 M_{\Lambda_{b}} .
\end{array}
$$

The factor of $\sqrt{2}$ in $\delta_{V}^{i}$ is a typical value and one may take a larger value to reduce the LD contributions in the regions of $I$ and $V$. The estimations of the decay branching ratios in the different regions are listed in Table 3. From the table, We find that the RE in region I is about $20 \%$ for the $e^{+} e^{-}$and $\mu^{+} \mu^{-}$modes and $25 \%$ for $\tau^{+} \tau^{-}$. The larger RE for the $\tau$ pair arises from $\Gamma_{4}$ in Eq. (12), which is proportional to the lepton mass. Moreover, this term also yields different distributions between the electron (or muon) and tau modes in region I when a large deviation from $\left(\left|C_{9}\left(m_{b}\right)\right|-\left|C_{10}\right|\right)$ appears. Therefore, studying the region with lower RE could distinguish the SD Wilson coefficients from the standard model.

Table 3: Decay branching ratios for QCD sume rule (SR) and Pole model (PM) with and without LD in different regions of $\Lambda$ energy with $\kappa=-1 /\left(3 C_{1}+C_{2}\right)$.

\begin{tabular}{|r|c|c|c|c|c|c|c|c|c|c|}
\hline \multirow{3}{*}{ Mode } & \multicolumn{10}{|c|}{$\mathrm{Br}$} \\
\cline { 2 - 12 } & I & \multicolumn{1}{|c|}{ II $\left(\times 10^{2}\right)$} & \multicolumn{1}{l|}{ III } & \multicolumn{1}{|c|}{ IV $\left(\times 10^{2}\right)$} & V & \\
\cline { 2 - 12 } & SR & PM & SR & PM & SR & PM & SR & PM & SR & PM \\
\hline \multirow{2}{*}{$e e$, LD } & 2.7 & 4.0 & 2.7 & 2.3 & 3.9 & 2.6 & 2.4 & 1.2 & 19.6 & 6.6 \\
\cline { 2 - 12 } NLD & 3.4 & 4.9 & 0.005 & 0.004 & 3.8 & 2.5 & 0.003 & 0.001 & 14.6 & 4.4 \\
\hline$\mu \mu$, LD & 2.7 & 4.0 & 2.7 & 2.3 & 3.9 & 2.6 & 2.4 & 1.2 & 17.9 & 6.2 \\
\cline { 2 - 11 } NLD & 3.4 & 4.9 & 0.005 & 0.004 & 3.8 & 2.5 & 0.003 & 0.001 & 12.9 & 4.0 \\
\hline$\tau \tau$, LD & 1.2 & 1.9 & 1.0 & 0.9 & 0.2 & 0.1 & -- & -- & -- & -- \\
\cline { 2 - 11 } NLD & 1.6 & 2.4 & 0.001 & 0.001 & 1.1 & 0.08 & -- & -- & -- & -- \\
\hline
\end{tabular}

As we can see from Eq. (3), the LD effects have been absorbed into the Wilson coefficient of $C_{9}^{e f f}$ and they are parametrized in the form of the phenomenological Breit-Wigner Ansatz. To compensate FA and VMD approximation, one phenomenological factor $\kappa$ is also introduced. In Table 4 , we show the decay branching ratios by taking $\kappa=-3.5$ and -1.9 . It is easily seen that in the regions of $I$ and $V$ the differences for the branching ratios with lower and higher $\kappa$ are between $5 \%-16 \%$. This tells us that, as expected, the uncertainty from the LD effect is small outside the resonance region.

In order to study how the effects arising from new physics beyond the standard model will affect the baryonic dilepton decays, we consider cases where the Wilson coefficients are different from those in the standard model. The results for the distributions of the differential branching rates are shown in Figures $3-6$. 
Table 4: Decay branching ratios in the whole range of $\Lambda$ energy including LD with two different values of $\kappa$.

\begin{tabular}{|c|c|c|c|c|c|}
\hline \multirow{2}{*}{ Decay Mode } & \multicolumn{5}{|c|}{$\mathrm{Br}$} \\
\cline { 2 - 6 } & $\mathrm{I}$ & $\mathrm{II}$ & $\mathrm{III}$ & $\mathrm{IV}$ & $\mathrm{V}$ \\
\hline$\Lambda_{b} \rightarrow \Lambda e^{+} e^{-}, \kappa=-3.5$ & 2.6 & $5.7 \times 10^{2}$ & 4.9 & $5.0 \times 10^{2}$ & 23.2 \\
\hline$\kappa=-1.9$ & 2.8 & $1.7 \times 10^{2}$ & 3.7 & $1.5 \times 10^{2}$ & 18.3 \\
\hline$\Lambda_{b} \rightarrow \Lambda \mu^{+} \mu^{-}, \kappa=-3.5$ & 2.6 & $5.7 \times 10^{2}$ & 4.9 & $5.0 \times 10^{2}$ & 21.4 \\
\hline$\kappa=-1.9$ & 2.8 & $1.7 \times 10^{2}$ & 3.7 & $1.5 \times 10^{2}$ & 16.5 \\
\hline$\Lambda_{b} \rightarrow \Lambda \tau^{+} \tau^{-}, \kappa=-3.5$ & 1.1 & $2.2 \times 10^{2}$ & 0.2 & -- & -- \\
\hline$\kappa=-1.9$ & 1.2 & $0.7 \times 10^{2}$ & 0.2 & -- & -- \\
\hline
\end{tabular}

We now discuss our results as follows:

- According to the results in Table 3 and Figures 1 and 2, we clearly see that outside the resonant regions the uncertainties arising from the QCD models are larger than that from the LD effects.

- We first compare our results in baryon decays with those in the $\mathrm{B}$ meson dilepton ones of $B \rightarrow K^{*} l^{+} l^{-}$[5, 6, 7, 8, 9, 10. In the meson decays, the pole of $\hat{s}$ is related to $\left|\hat{m}_{b} C_{7} / \hat{s}\right|^{2}$ and $\hat{m}_{b} C_{7} / \hat{s}$, respectively, and thus with the requirement $\hat{s} \geq 4 \hat{m}_{l}$ from the phase space, the processes of $B \rightarrow K^{*} \mu^{+} \mu^{-}$and $B \rightarrow K^{*} e^{+} e^{-}$have very different decay rates. However, for the decays of $\Lambda_{b} \rightarrow \Lambda l^{+} l^{-}$, the associated terms are proportional to $\left|\hat{m}_{l} \hat{m}_{b} C_{7}\right|^{2} / \hat{s}^{2}$ and $\left|\hat{m}_{b} C_{7}\right|^{2} / \hat{s}$. Clearly, due to the mass suppression for the light lepton, the main pole dependence is $\sim\left|\hat{m}_{b} C_{7}\right|^{2} / \hat{s}$ so that the rate difference between $\Lambda_{b} \rightarrow \Lambda \mu^{+} \mu^{-}$and $\Lambda_{b} \rightarrow \Lambda e^{+} e^{-}$is small.

- The differential decay rates of $\Lambda_{b} \rightarrow \Lambda l^{+} l^{-}$are sensitive to the signs of $C_{9}$ and $C_{7}$. Although $C_{7} \ll C_{9}$ and $C_{10}$, there exists an enhanced factor of $12 \operatorname{Re} C_{9} C_{7}^{*} \sim 15.3$ in $\Gamma_{2}$ of Eq. (12). When the sign of $C_{7}$ is opposite to that in the standard model, there is a deviation of $50 \%$ in branching ratio for neglecting RE. Thus, the contribution from electromagnetic part cannot be neglected. As changing the sign of $C_{9}$ to be opposite to that in the standard model, only a deviation of $27 \%$ on the branching ratios occurs. However, from Figures 3 and 5 , we see that the distributions are different from each others.

- From Eq. (12), we find that the differential decay rates cannot have the information in the sign of $C_{10}$ since they are always related to $\left|C_{10}\right|^{2}$.

- From Figures 4 and 6 , we find that with $C_{10}=\left.2 C_{9}\right|_{S M}$ the distribution for the differential decay rate of the $\tau^{+} \tau^{-}$mode is higher than that with $C_{9}=\left.2 C_{10}\right|_{S M}$ in region I but it is reversed in that of the $\mu^{+} \mu^{-}$distribution. The origin of this difference is from the $\Gamma_{4}$ in Eq. ( 12) which is proportional to $6 \hat{m}_{l}^{2}\left(\left|C_{9}\right|^{2}-\left|C_{10}\right|^{2}\right)$. This effect can be neglected in the standard model since $\left|C_{9}\right| \sim\left|C_{10}\right|$ and the light lepton modes as well. Although $\hat{m}_{\tau}^{2} \sim 10 \%$, this factor will become important. if there is a large deviation between $C_{9}$ and $C_{10}$.

- The decay width distributions for the longitudinal polarized $\Lambda$ with and without LD effects as the function of $\Lambda$ energy are shown in Figures 7 and 8. Comparing the figures with the differential decay branching rates in Figures 1 and 2, respectively, we find that the distributions are very similar to each other except the opposite sign. 
- Finally, as usual, from Eq. (9) we may also write the partial decay rate as

$$
d \Gamma_{\Lambda_{b}}=\frac{1}{2} \Gamma_{0}\left(1-\alpha_{\Lambda} \hat{\mathrm{p}} \cdot \hat{\mathrm{s}} d \cos \theta_{\Lambda}\right),
$$

where $\Gamma_{0}$ is related to the decay width of $\Lambda_{b} \rightarrow \Lambda l^{+} l^{-}, \hat{\mathrm{p}}$ is the unit direction of $\Lambda$ momentum in the $\Lambda_{b}$ rest frame, and $\alpha_{\Lambda}$, called $\Lambda$ polarization, is defined by

$$
\alpha_{\Lambda}=\frac{\int_{r}^{t_{\max }} D_{L}\left(1-4 \hat{m}_{l}^{2} / \hat{s}\right)\left(\hat{t}^{2}-r\right) / \sqrt{r} d \hat{t}}{\int_{r}^{t_{\max }} \sqrt{1-4 \hat{m}_{l}^{2} / \hat{s}} \sqrt{\hat{t}^{2}-r} \rho_{0}(\hat{t}) d \hat{t}},
$$

where $t_{\max }=\left(1+r-4 \hat{m}_{l}^{2}\right) / 2$. Numerically, we find that the polarizations of $\Lambda$ in $\Lambda_{b} \rightarrow$ $\Lambda l^{+} l^{-}(l=e, \mu, \tau)$ decays are all unity, $\alpha_{\Lambda} \approx 1$.

\subsection{Polarization asymmetries}

In this subsection we will discuss longitudinal and normal polarization asymmetries and their implications and we will study the transverse polarization elsewhere [15] since it is zero in the standard model as mentioned in Sec. 3. From Eq. (14), we show the the distributions of $P_{L}$ and $P_{N}$ with respect to the dimensionless kinematic variable $\hat{t}$ in Figures $9-12$, respectively. From the figures, we find the following interesting results:

- The polarization asymmetries are insensitive to the LD effects.

- The values of $P_{L}$ are near unity except a narrow region with a small $\Lambda$ momentum.

- $P_{N}$ approaches zero as the $\Lambda$ energy increases. This is because the polarization is proportional to $\sqrt{\hat{s}}$ as shown in Eq. (16).

- The values of $P_{L, N}$ from the QCD sum rule and the pole models shown in the figures are close to each other. The results imply that both $P_{L}$ and $P_{N}$ are not very sensitive to the form factors. Therefore, one would like to use $P_{L, N}$ to probe the short-distance (SD) physics due to the smallness of the uncertainties from the strong interaction.

We now discuss the sensitivity for the longitudinal polarization of $P_{L}$ to new physics. We first notice that by using different values of the Wilson coefficients from the standard model, the polarizations do not change. The reason is that the coefficients get canceled out between the denominator and numerator in Eq. (14). However, in our derivation for the differential decay rate, we have assumed the $V-A$ hadronic current and neglected the contribution of left-handed electromagnetic moment since it is proportional to the strange quark mass. If we include the interaction with the right-handed current, the polarization will behave quit different from that in the standard model, which can be understand easily by Eq. (26) of Ref. [12] as $h_{A} \neq h_{V}$. Finally, we define the integrated longitudinal and normal polarization asymmetries as

$$
\begin{aligned}
& \bar{P}_{L}=\int d \hat{t} P_{L}, \\
& \bar{P}_{N}=\int d \hat{t} P_{N} .
\end{aligned}
$$

In the standard model, we obtain that $\bar{P}_{L(N)}=-0.31(0.02)$ and $\bar{P}_{L(N)}=-0.12(0.01)$ for $\mu \mu$ and $\tau \tau$ modes, respectively. If deviations from the standard model predictions for the integrated polarization asymmetries are measured, it is clear that there exit some kinds of new physics. 


\section{Conclusions}

We have studied the rare baryonic exclusive decays of $\Lambda_{b} \rightarrow \Lambda l^{+} l^{-}(l=e, \mu, \tau)$ with polarized $\Lambda$. Under the approximation of HQET, in the standard model we have derived the differential decay rates and the polarization asymmetries of $\Lambda$ by including lepton mass effects.

We have found that with the LD effects the decay branching ratios of $\Lambda_{b} \rightarrow \Lambda l^{+} l^{-}(l=$ $e, \mu, \tau)$ are $5.3 \times 10^{-5}, 5.3 \times 10^{-5}$, and $1.1 \times 10^{-5}$ from the QCD sum rule approach and $1.2 \times 10^{-5}, 1.2 \times 10^{-5}$, and $3.2 \times 10^{-6}$ from the the pole model, respectively. We have also estimated the decay branching ratio of $\Lambda_{b} \rightarrow \Lambda \nu \bar{\nu}$ to be $1.6 \times 10^{-5}$ and $3.3 \times 10^{-6}$ in the two models, respectively. In physics beyond the standard model, we have studied various cases of different Wilson coefficients. We have shown that the decay rates as well as the distributions can be very different from those in the standard model.

The integrated longitudinal $\Lambda$ polarizations are -0.31 and -0.12 , while that of the normal ones 0.02 and 0.01 , for di-muon and tau modes, respectively. The CP-odd transverse polarization of $\Lambda$ is zero in the standard model but it is expected to be sizable in new physics such as the CP violating theories with right-handed interactions. We have demonstrated that the polarization asymmetries are insensitive to LD contributions but sensitive to the right-handed couplings. It is clear that one could probe new physics through measurements of the $\Lambda$ polarizations in the decays of $\Lambda_{b} \rightarrow \Lambda l^{+} l^{-}$.

\section{Acknowledgments}

This work was supported in part by the National Science Council of the Republic of China under contract numbers NSC-89-2112-M-007-054 and NSC-89-2112-M-006-033. 


\section{References}

[1] CLEO Collaboration, M. S. Alam et. al., Phys. Rev. Lett. 74, 2885 (1995).

[2] N. Cabibbo, Phys. Rev. Lett. 10, 531 (1963); M. Kobayashi and T. Maskawa, Prog. Theor. Phys. 49, 652 (1973).

[3] For a recent review, see A. Ali et. al., Phys. Rev. D61, 074024 (2000).

[4] G. Buchalla, A. J. Buras and M. E. Lautenbacher, Rev. Mod. Phys. 68, 1230 (1996).

[5] N.G. Deshpande, J. Trampetic and K. Panose, Phys. Rev. D39, 1462 (1989).

[6] C.S. Lim, T. Morozumi, and A.T. Sanda, Phys. Lett. B218, 343 (1989).

[7] A. Ali, T. Mannel, and T. Morozumi, Phys. Lett. B273, 505 (1991).

[8] P.J. O'Donnell and K.K.K. Tung, Phys. Rev. D43, R2067 ( 1991).

[9] F. Krüger and L.M. Sehgal, Phys. Lett. B380, 199 (1996).

[10] C.Q. Geng and C. P. Kao, Phys. Rev. D54, 5636 (1996).

[11] T. Mannel, W. Roberts and Z. Ryzak Nucl. Phys. B355, 38 (1991).

T. Mannel and S. Recksiegel J. Phys. G24, 979 (1998).

[12] Chao-Shang Huang and Hua-Gang Yan, Phys Rev. D59, 114022 (1999).

[13] T.M. Aliev and M. Savci, J. Phys. G26, 997 (2000).

[14] CLEO Collaboration,G. Crawford et al Phys. Rev. Lett. 75, 624 (1995).

[15] Chuan-Hung Chen and C. Q. Geng, in progress. 


\section{Figure Captions}

Figure 1: The differential decay branching ratio of $\Lambda_{b} \rightarrow \Lambda \mu^{+} \mu^{-}$as a function of $\Lambda$ energy. The solid and dashed curves stand for the QCD sum rule and pole models, respectively.

Figure 2: $\quad$ Same as Figure 1 but for $\Lambda_{b} \rightarrow \Lambda \tau^{+} \tau^{-}$.

Figure 3: The differential decay branching ratio of $\Lambda_{b} \rightarrow \Lambda \mu^{+} \mu^{-}$as a function of $\Lambda$ energy with the Wilson coefficients being different from those in the standard model. The solid, dashed, dotted, long-dashed, and dash-dotted curves represent the results of the standard model $(\mathrm{SM}), C_{10}=0, C_{9}=-\left.C_{9}\right|_{S M}, C_{7}=-\left.C_{7}\right|_{S M}$, and $C_{7}=0$, respectively.

Figure 4: $\quad$ Same as Figure 3 but the dashed, dotted, long-dashed, and dash-dotted curves are for $C_{9}=-\left.2 C_{9}\right|_{S M}, C_{9}=-\left.2 C_{9}\right|_{S M}$ and $C_{7}=0, C_{9}=\left.2 C_{9}\right|_{S M}$, and $C_{10}=$ $\left.2 C_{10}\right|_{S M}$, respectively.

Figure 5: $\quad$ Same as Figure 3 but for $\Lambda_{b} \rightarrow \Lambda \tau^{+} \tau^{-}$.

Figure 6: $\quad$ Same as Figure 4 but for $\Lambda_{b} \rightarrow \Lambda \tau^{+} \tau^{-}$.

Figure 7: The decay width distribution of $\Lambda_{b} \rightarrow \Lambda \mu^{+} \mu^{-}$for the longitudinal polarized $\Lambda$ as a function of $\Lambda$ energy with and without $\mathrm{RE}$.

Figure 8: $\quad$ Same as Figure 7 but for $\Lambda_{b} \rightarrow \Lambda \tau^{+} \tau^{-}$.

Figure 9: The longitudinal polarization asymmetry of $\Lambda_{b} \rightarrow \Lambda \mu^{+} \mu^{-}$as a function of $E_{\Lambda} / M_{\Lambda}$. Legend is the same as Figure 1.

Figure 10: Same as Figure 9 but for $\Lambda_{b} \rightarrow \Lambda \tau^{+} \tau^{-}$.

Figure 11: The normal polarization asymmetry of $\Lambda_{b} \rightarrow \Lambda \mu^{+} \mu^{-}$as a function of $E_{\Lambda} / M_{\Lambda}$. Legend is the same as Figure 1.

Figure 12: Same as Figure 11 but for $\Lambda_{b} \rightarrow \Lambda \tau^{+} \tau^{-}$. 


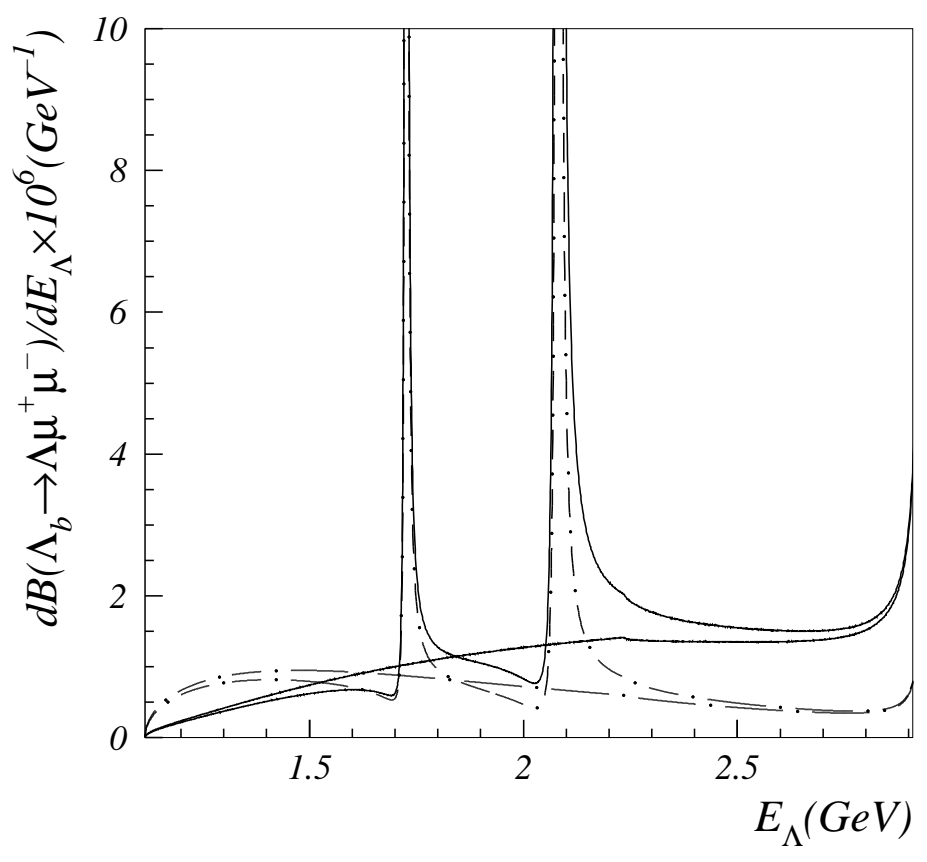

Figure 1: The differential decay branching ratio of $\Lambda_{b} \rightarrow \Lambda \mu^{+} \mu^{-}$as a function of $\Lambda$ energy. The solid and dashed curves stand for the QCD sum rule and pole models, respectively. 


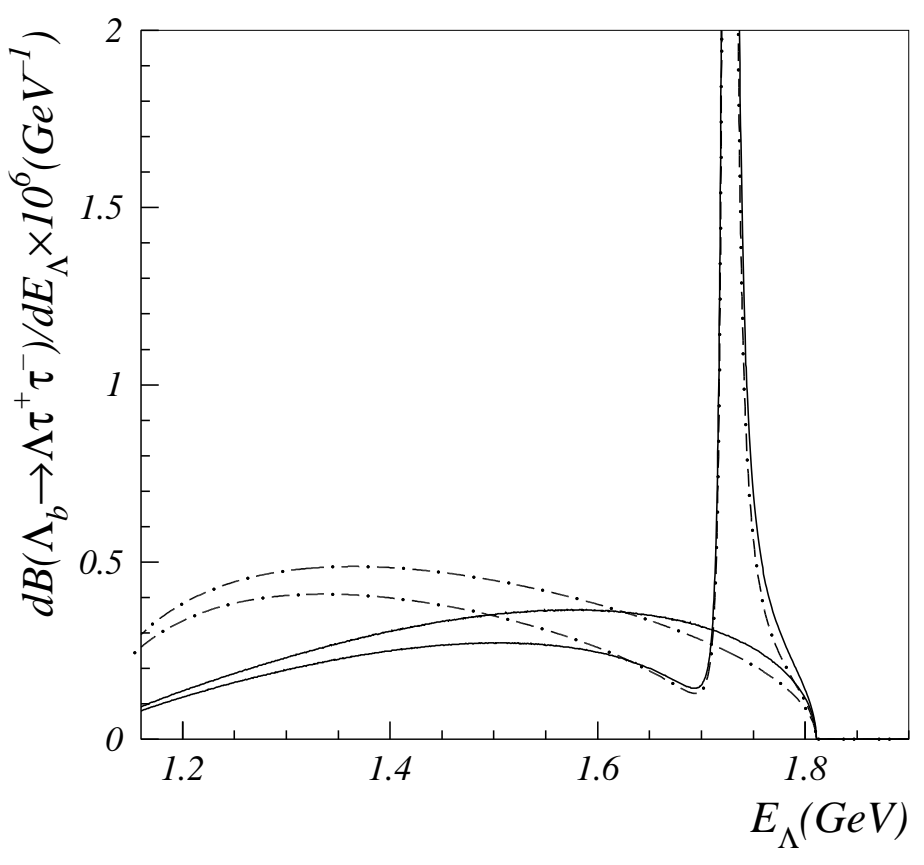

Figure 2: Same as Figure 1 but for $\Lambda_{b} \rightarrow \Lambda \tau^{+} \tau^{-}$. 


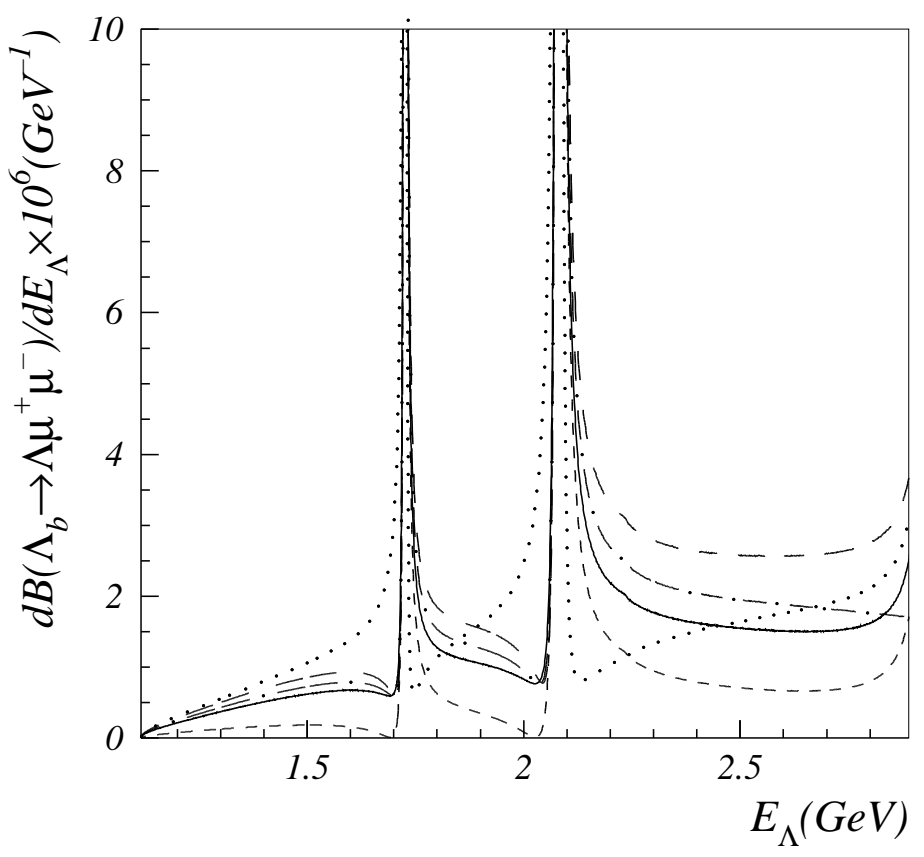

Figure 3: The differential decay branching ratio of $\Lambda_{b} \rightarrow \Lambda \mu^{+} \mu^{-}$as a function of $\Lambda$ energy with the Wilson coefficients being different from those in the standard model. The solid, dashed, dotted, long-dashed, and dash-dotted curves represent the results of the standard model (SM), $C_{10}=0, C_{9}=-\left.C_{9}\right|_{S M}, C_{7}=-\left.C_{7}\right|_{S M}$, and $C_{7}=0$, respectively. 


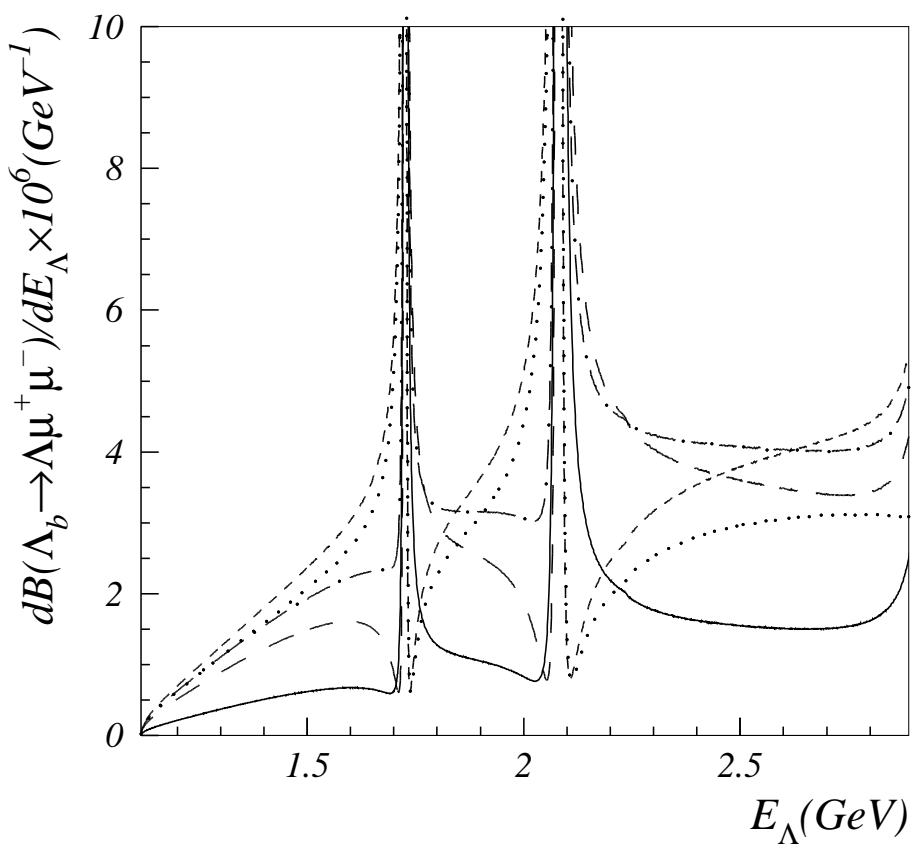

Figure 4: Same as Figure 3 but the dashed, dotted, long-dashed, and dash-dotted curves are for $C_{9}=-\left.2 C_{9}\right|_{S M}, C_{9}=-\left.2 C_{9}\right|_{S M}$ and $C_{7}=0, C_{9}=\left.2 C_{9}\right|_{S M}$, and $C_{10}=\left.2 C_{10}\right|_{S M}$, respectively. 


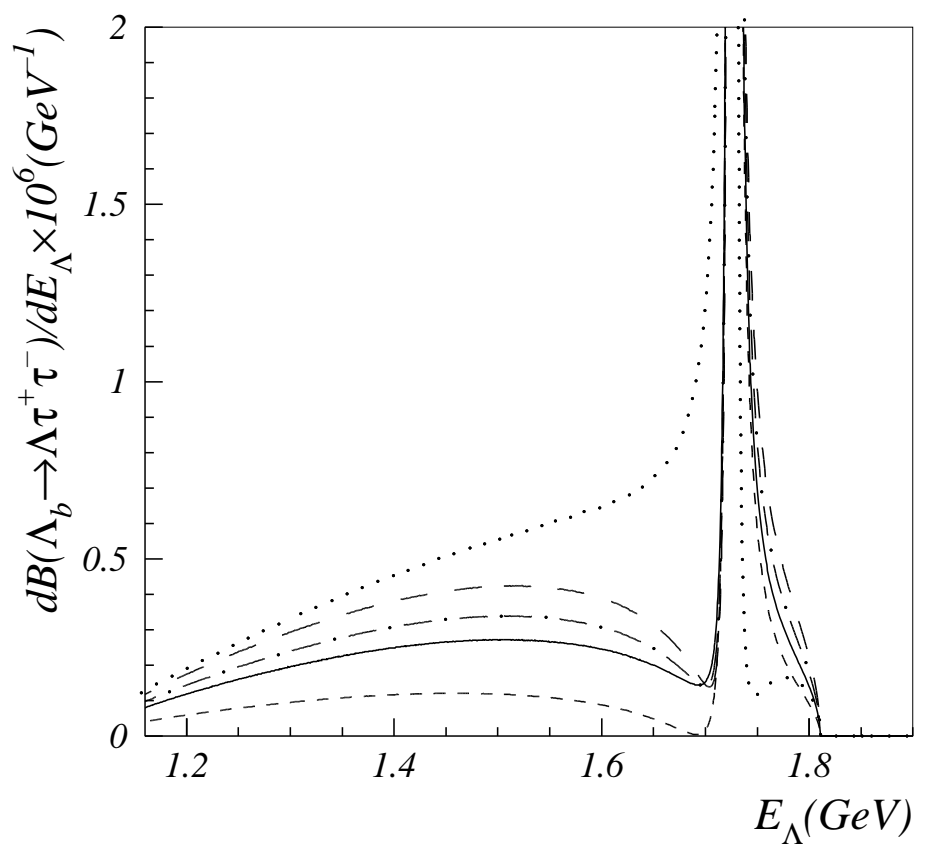

Figure 5: Same as Figure 3 but for $\Lambda_{b} \rightarrow \Lambda \tau^{+} \tau^{-}$. 


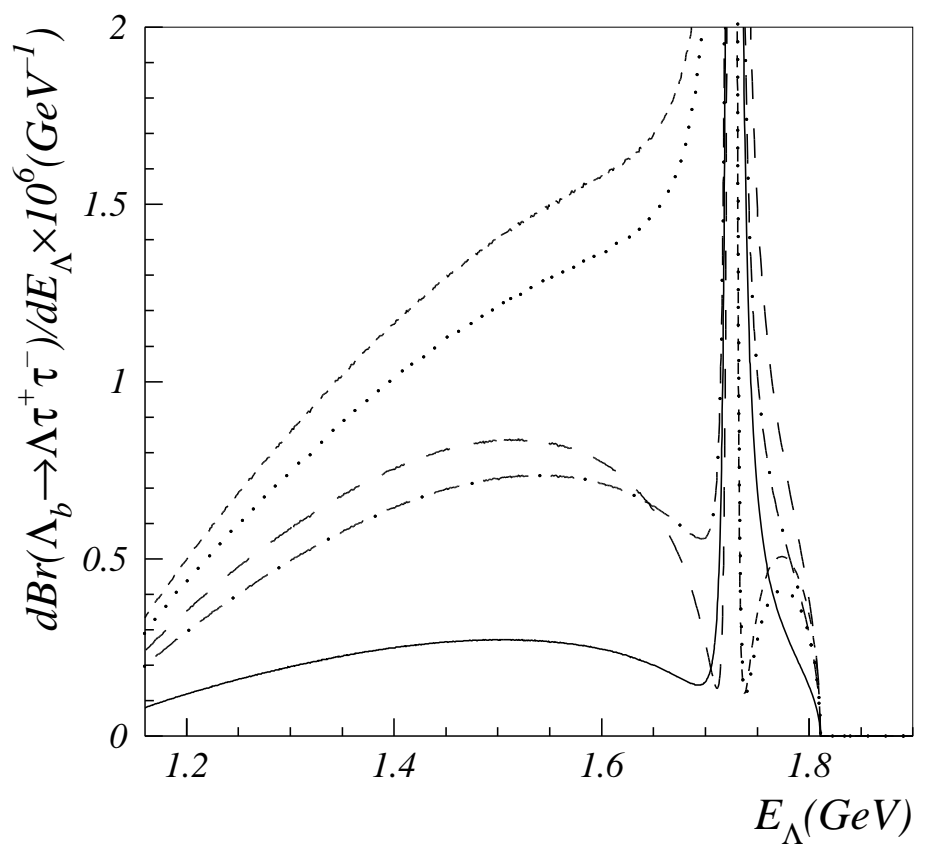

Figure 6: Same as Figure 4 but for $\Lambda_{b} \rightarrow \Lambda \tau^{+} \tau^{-}$. 


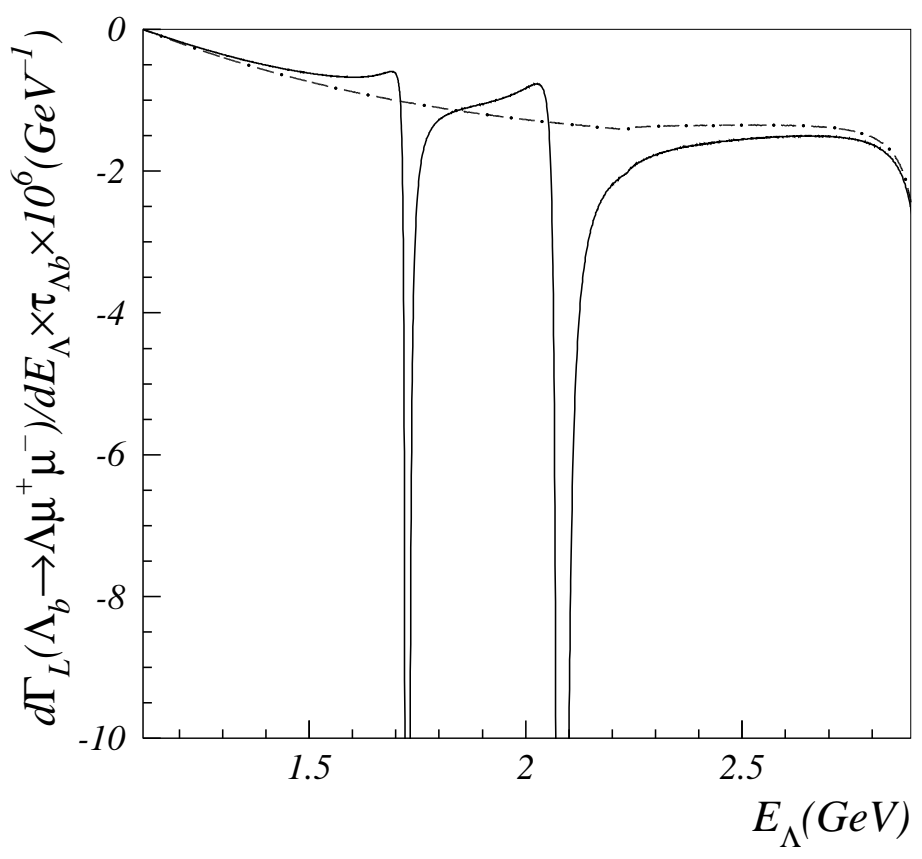

Figure 7: The decay width distribution of $\Lambda_{b} \rightarrow \Lambda \mu^{+} \mu^{-}$for the longitudinal polarized $\Lambda$ as a function of $\Lambda$ energy with and without RE. 


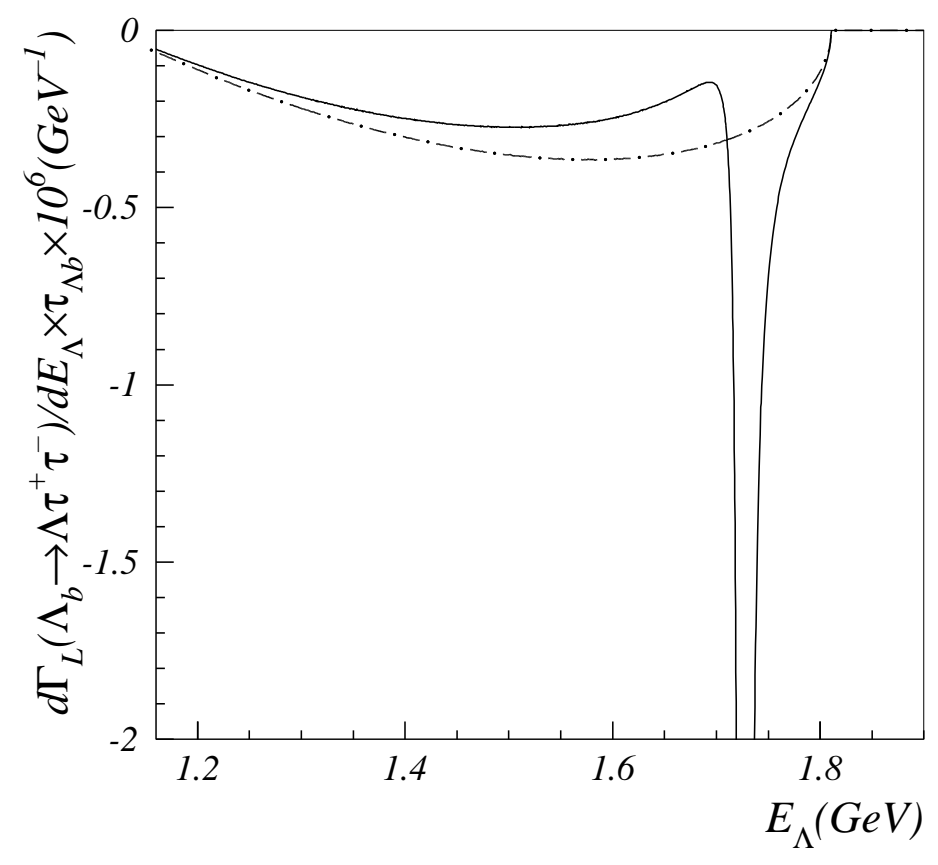

Figure 8: Same as Figure 7 but for $\Lambda_{b} \rightarrow \Lambda \tau^{+} \tau^{-}$. 


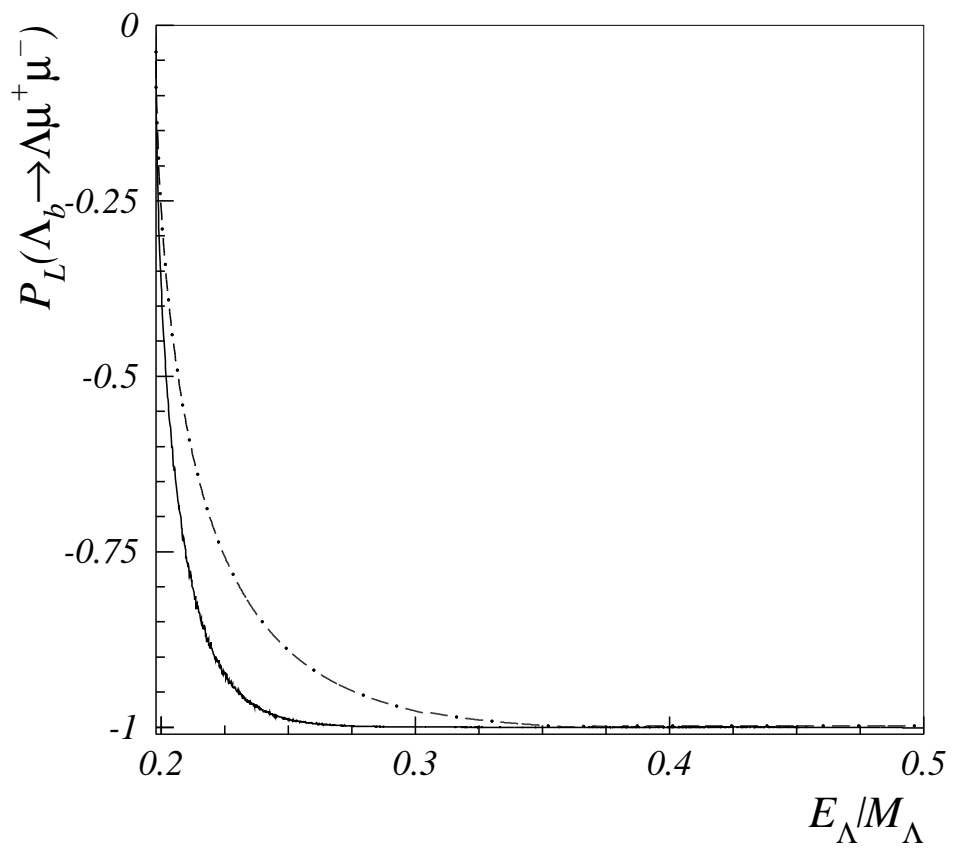

Figure 9: The longitudinal polarization asymmetry of $\Lambda_{b} \rightarrow \Lambda \mu^{+} \mu^{-}$as function of $E_{\Lambda} / M_{\Lambda}$. Legend is the same as Figure 1. 


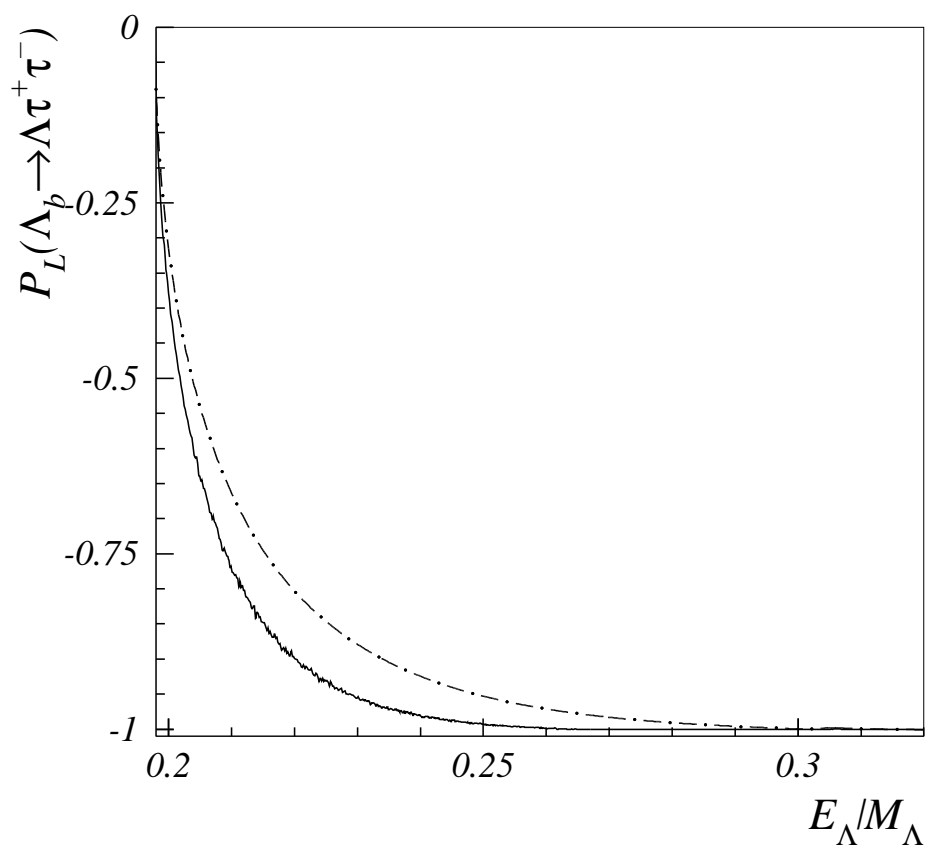

Figure 10: Same as Figure 9 but for $\Lambda_{b} \rightarrow \Lambda \tau^{+} \tau^{-}$. 


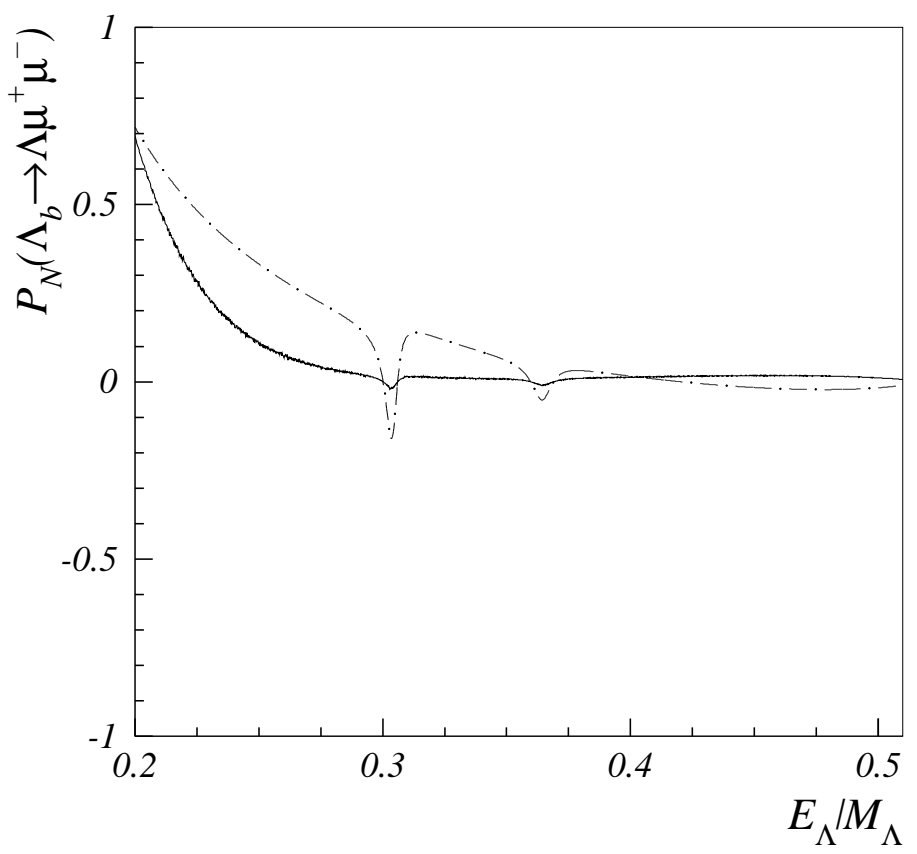

Figure 11: The normal polarization asymmetry of $\Lambda_{b} \rightarrow \Lambda \mu^{+} \mu^{-}$as function of $E_{\Lambda} / M_{\Lambda}$. Legend is the same as Figure 1. 


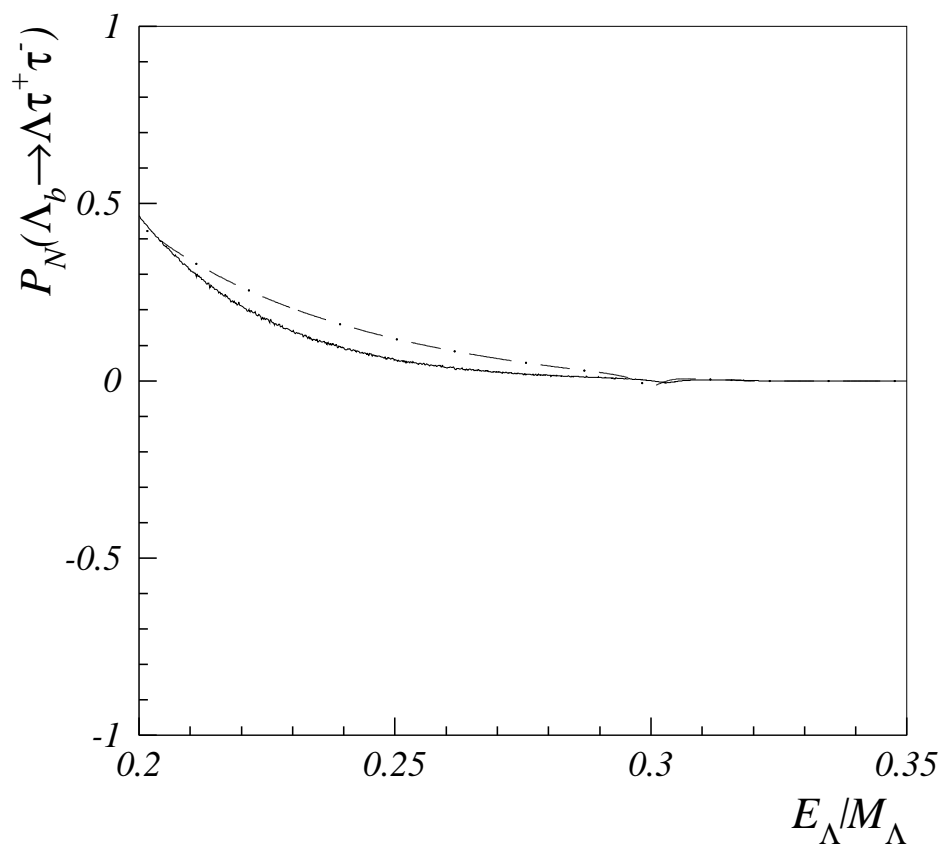

Figure 12: Same as Figure 11 but for $\Lambda_{b} \rightarrow \Lambda \tau^{+} \tau^{-}$. 